\title{
Treatment of refractory/relapsed adult acute lymphoblastic leukemia with bortezomib- based chemotherapy
}

This article was published in the following Dove Press journal:

International Journal of General Medicine

12 June 2015

Number of times this article has been viewed

\author{
Junmei Zhao* \\ Chao Wang* \\ Yongping Song \\ Yuzhang Liu \\ Baijun Fang
}

Henan Key Lab of Experimental Haematology, Henan Institute of Haematology, Henan Tumor Hospital, Zhengzhou University, Zhengzhou, People's Republic of China

*These authors contributed equally to this work
Correspondence: Baijun Fang Henan Key Lab of Experimental Haematology, Henan Institute of Haematology, Henan Tumor Hospital, Zhengzhou University, 127 Dongming Road, Zhengzhou 450008, People's Republic of China Tel +86 37l 65587328 Fax +86 37I 65587796 Email sunfbj@126.com
Abstract: Nine pretreated patients aged $>19$ years with relapsed/refractory acute lymphoblastic leukemia (ALL) were treated with a combination of bortezomib plus chemotherapy before allogeneic hematopoietic stem cell transplantation (allo-HSCT). Eight (88.9\%) patients, including two Philadelphia chromosome-positive ALL patients, achieved a complete remission. Furthermore, the evaluable patients have benefited from allo-HSCT after response to this reinduction treatment. We conclude that bortezomib-based chemotherapy was highly effective for adults with refractory/relapsed ALL before allo-HSCT. Therefore, this regimen deserves a larger series within prospective trials to confirm these results.

Keywords: acute lymphoblastic leukemia, refractory, relapsed, bortezomib

\section{Introduction}

The prognosis in patients with refractory/relapsed adult acute lymphoblastic leukemia (ALL) is dismal. Currently, there is no standard salvage therapy for such patients and the only realistic strategy resides in getting another complete remission (CR) followed by a successful allogeneic hematopoietic stem cell transplantation (allo-HSCT), ${ }^{1-3}$ provided that the toxicity of the salvage regimen is acceptable. In the reinduction setting, the use of chemotherapeutic agents similar to those administered during initial induction chemotherapy may get $\mathrm{CR}$, but the possibility of achieving a second $\mathrm{CR}$ is less than $50 \% .{ }^{4}$ In addition, this regimen is limited, particularly in adults with refractory ALL. Treatment strategies to further improve the efficacy of antileukemic therapy are often based on the introduction of novel agents. Recently, Dewar et $\mathrm{al}^{5}$ reported that a patient diagnosed with refractory adult Philadelphia chromosome-positive $\left(\mathrm{Ph}^{+}\right) \mathrm{ALL}$ was treated successfully with a bortezomib-containing chemotherapy regimen. In addition, Hu et $\mathrm{al}^{6}$ obtained encouraging results in a relapsed adult T-cell acute lymphoblastic leukemia (T-ALL) by administration of bortezomib combined with chemotherapy. Thus far, no series has reported the use of bortezomib for the treatment of relapsed/refractory adult ALL. We report here the outcomes of nine such patients treated by a combination of bortezomib plus chemotherapy as part of a pilot study. Favorable responses were observed.

\section{Case report}

Between March 2008 and November 2010, a total of nine consecutive patients with refractory/relapsed adult $\mathrm{ALL}$, including $\mathrm{Ph}^{+} \mathrm{ALL}$, were enrolled in this study at the Henan Institute of Haematology (Table 1). Informed consent was obtained from all patients and the study was approved by the Institutional Review Board at 


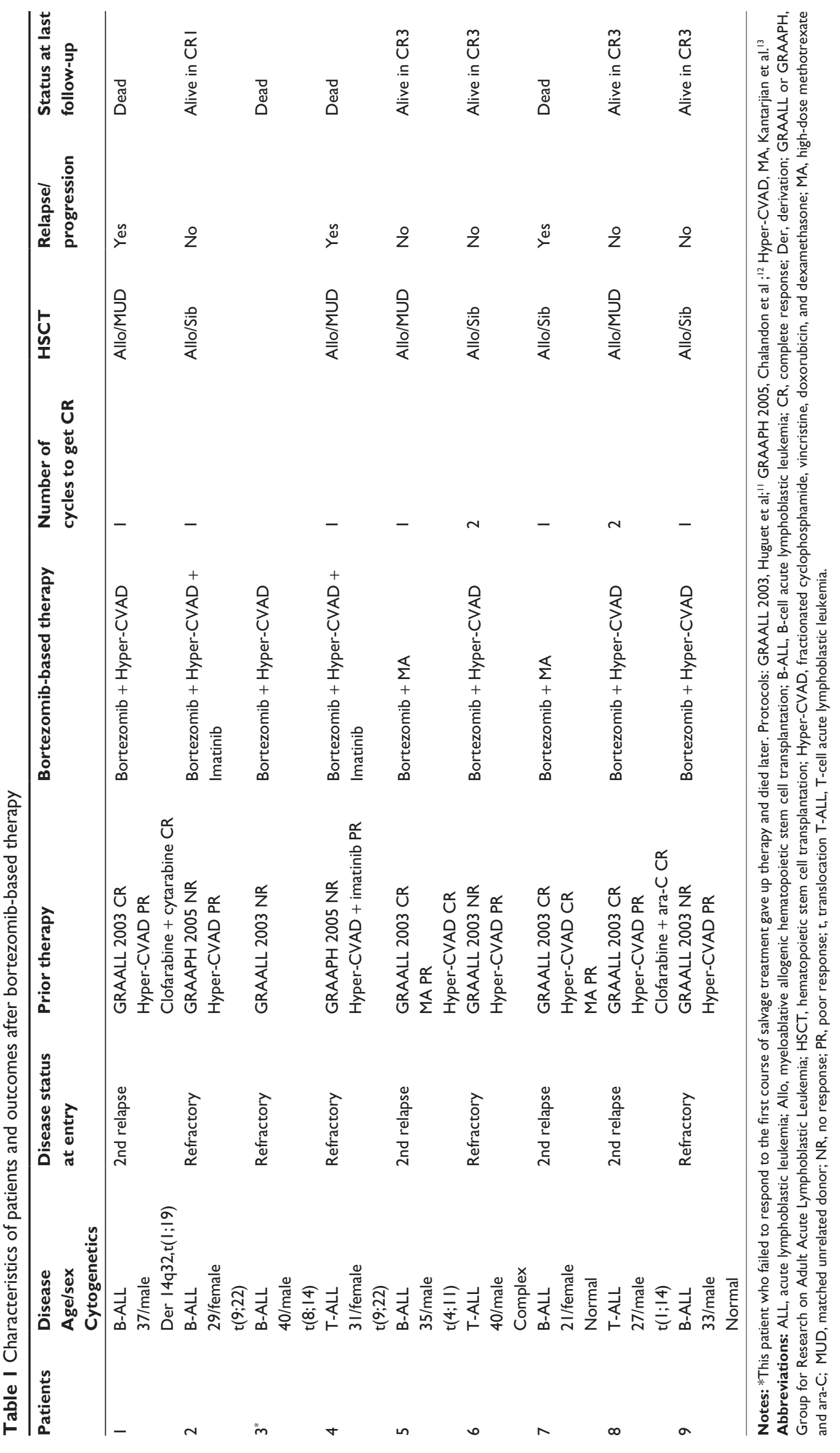


Henan Tumor Hospital. Other eligibility criteria included a performance status of 2 or lower according to the Eastern Cooperative Oncology Group (ECOG), adequate hepatic and renal function, and adequate cardiac status.

As a salvage reinduction regimen, all patients received a bortezomib-based therapy at a median time of 6 months (range 1.9-13.5 months) after ALL diagnosis. Five patients received the bortezomib $\left(1.3 \mathrm{mg} / \mathrm{m}^{2} /\right.$ day on days $1,4,8$, and 11$)+$ fractionated cyclophosphamide, vincristine, doxorubicin, and dexamethasone (Hyper-CVAD) regimen and two patients received the bortezomib $\left(1.3 \mathrm{mg} / \mathrm{m}^{2} /\right.$ day on days $1,4,8$, and 11) + (high-dose methotrexate and ara-C) regimen. The other two patients with refractory $\mathrm{Ph}^{+} \mathrm{ALL}$ were treated with bortezomib $\left(1.3 \mathrm{mg} / \mathrm{m}^{2} /\right.$ day on days $1,4,8$, and 11$)+$ imatinib (400 mg/day) + Hyper-CVAD regimen. CR was defined as $\leq 5 \%$ lymphoblasts in a normocellular or hypercellular marrow with no evidence of circulating blasts or extramedullary disease, and with absolute neutrophil count $1 \times 10^{9} / \mathrm{L}$ and platelet count $\geq 100 \times 10^{9} / \mathrm{L}$. Treatment-related toxicities were assessed and graded according to the National Cancer Institute Expanded Common Toxicity Criteria (version 3.0). After a salvage reinduction regimen, patients getting $\mathrm{CR}$ could receive one or more first consolidation courses, followed by allo-HSCT as soon as possible. After allo-HSCT, patients with refractory/relapsed $\mathrm{Ph}^{+} \mathrm{ALL}$ were maintained on dasatinib (70 mg twice a day). Patients who failed to obtain CR after two courses were taken off the study. A third-line regimen called FLAG-IDA was suggested for patients who failed to obtain CR after the salvage treatment and for those who relapsed after the salvage treatment and before allo-HSCT. ${ }^{7}$ Overall survival (OS) was calculated from the beginning of salvage therapy to the date of the last follow-up or death for survivors, whatever the cause, while disease-free survival (DFS) was calculated from CR until relapse, death from any cause, or the last contact for survivors. Probability of OS rate was estimated according to the Kaplan-Meier method.

Table 1 shows the responses and outcome details of therapy. Patient 3, who failed to respond to the first course of salvage treatment, gave up therapy and died 1.7 months after stopping treatment. The other eight patients achieved CR after 1-2 courses of bortezomib-containing chemotherapy. Then, after receiving one or more first consolidation courses, all underwent an allo-HSCT. At the time of the last follow-up, three of eight eligible patients who had achieved CR died of leukemia relapse (at 4.2, 10.6, and 13.8 months, respectively), while the other five patients were still alive and in CR at 15.1, 16.3, 35.8, and 44.3 months, respectively. The median DFS was 15.7 months (range 8.1-44.3 months) and the median OS was 16.4 months (range 2.7-45.4 months). Two-year OS rate was 56.4 months (95\% CI 8.9-30.7 months) (Figure 1). The combination of bortezomib plus chemotherapy was well tolerated. No patient discontinued therapy because of toxicity and there was no treatment-related mortality. All patients experienced grade 3-4 anemia, neutropenia, and thrombocytopenia. The median time to reach absolute neutrophil count $>0.5 \times 10^{9} / \mathrm{L}$ was 19 days (range 15-24 days) from the start of chemotherapy. Platelet count $>20 \times 10^{9} / \mathrm{L}$ was achieved in a median time of 21 days (range 18-26 days). The most common grade 1-2 nonhematologic toxicities in the study included constipation (50\%), vomiting (100\%), fatigue (100\%), diarrhea (37.5\%), and skin rashes $(12.5 \%)$. In addition, Grade $1-2$ peripheral neuropathy and grade 3 pulmonary infections occurred in one (12.5\%) and four $(50 \%)$ patients, respectively.

\section{Discussion}

Treatment of refractory/relapsed adult ALL, including $\mathrm{Ph}^{+}$ ALL, represents a considerable clinical challenge. These patients are often refractory to currently available treatment options, and the alternative salvage therapeutic approaches remain elusive.

Single-agent bortezomib was studied in adults with refractory/relapsed acute leukemia and found to be ineffective in controlling disease progression, ${ }^{8}$ but the results from a combination trial have suggested bortezomib is a rational strategy to overcome chemoresistance and induce chemosensitization. Attar et $\mathrm{al}^{9}$ reported that the treatment of bortezomib plus cytarabine and idarubicin in patients with relapsed acute myeloid leukemia showed encouraging activity, and that the regimen had also been well tolerated. In addition, in patients with fludarabine-refractory chronic lymphocytic leukemia, single-agent bortezomib demonstrated biologic

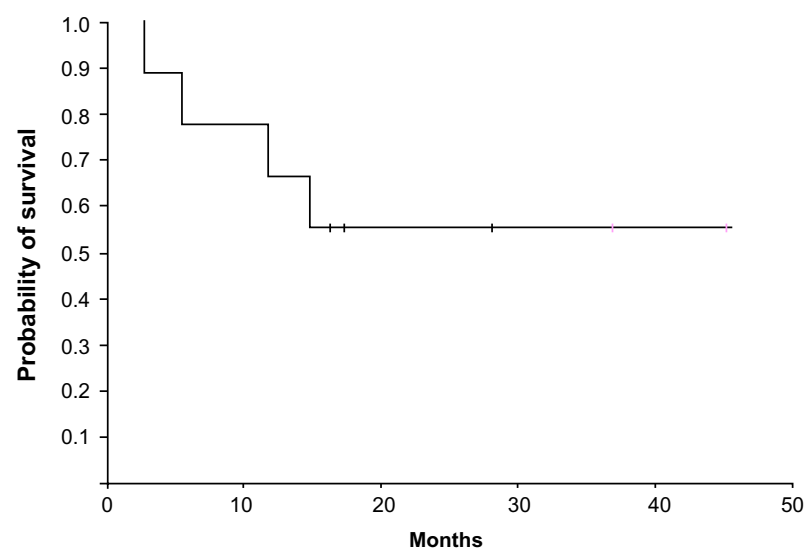

Figure I Overall survival in nine patients with refractory/relapsed adult acute lymphoblastic leukemia treated with bortezomib-based therapy. 
activity. ${ }^{10}$ Recently, Hu et $\mathrm{al}^{6}$ reported that administration of bortezomib plus dexamethasone and liposomal doxorubicin to a heavily pretreated adult with relapsed ALL achieved another CR.

Our results suggest that the addition of bortezomib to conventional chemotherapy programs in adults with refractory/ relapsed ALL is feasible and that the salvage treatment was generally well tolerated. Indeed, we can confidently conclude that CR achievement was due to the addition of bortezomib. All eight evaluable patients had refractory disease after receiving Hyper-CVAD-based or imatinib-based induction regimens, but they were all in $\mathrm{CR}$ after receiving the bortezomib + HyperCVAD, or bortezomib + Hyper-CVAD + imatinib, respectively, indicating the efficacy of bortezomib and a synergistic effect of the combination. Thus, the combined bortezomib-based reinduction therapy may be a promising salvage alternative for this population with a very poor prognosis as a springboard to allo-HSCT, the only potential curative intervention.

Furthermore, all eight eligible patients have benefited from allo-HSCT as consolidation after CR was assessed. However, the idea that bortezomib could overcome or reverse chemoresistance and increase sensitivity to conventional or targeted agents was not evaluated in this study. This could be a pivotal factor in selecting optimal patients who might respond to the combination of bortezomib plus conventional chemotherapy, and this should be addressed in future studies.

\section{Conclusion}

Reinduction with the bortezomib-based regimen followed by allo-HSCT may be a feasible approach for adults with refractory/relapsed ALL. Larger series within prospective trials are needed to evaluate this concept.

\section{Author contributions}

All authors designed the research, enrolled the study patients, supervised the study, collected clinical data, analyzed the data, and drafted and revised the manuscript.

\section{Acknowledgments}

The authors would like to thank all patients for their cooperation. This study was supported by grants from the
National Natural Science Foundation of China (Number 30900637) and the National Natural Science Foundation of China (Number 81370661).

\section{Disclosure}

The authors declare no potential conflicts of interest in this work.

\section{References}

1. Atra A, Gerrard M, Hobson R, Imeson JD, Hann IM, Pinkerton CR. Outcome of relapsed or refractory childhood B-cell acute lymphoblastic leukaemia and B-cell non-Hodgkin's lymphoma treated with the UKCCSG 9003/9002 protocols. Br J Haematol. 2001;112(4):965-968.

2. Martino R, Bellido M, Brunet S, et al. Allogeneic or autologous stem cell transplantation following salvage chemotherapy for adults with refractory or relapsed acute lymphoblastic leukemia. Bone Marrow Transplant. 1998;21(10):1023-1027.

3. Martino R, Bellido M, Brunet S, et al. Intensive salvage chemotherapy for primary refractory or first relapsed adult acute lymphoblastic leukemia: results of a prospective trial. Haematologica. 1999;84(6): 505-510.

4. Weiss MA. Treatment of adult patients with relapsed or refractory acute lymphoblastic leukemia (ALL). Leukemia. 1997;11 Suppl 4: S28-S30.

5. Dewar R, Chen ST, Yeckes-Rodin H, Miller K, Khosravi-Far R. Bortezomib treatment causes remission in a Ph+ ALL patient and reveals FoxO as a theranostic marker. Cancer Biol Ther. 2011;11(6):552-558.

6. Hu X, Xu J, Sun A, Shen Y, He G, Guo F. Successful T-cell acute lymphoblastic leukemia treatment with proteasome inhibitor bortezomib based on evaluation of nuclear factor- $\mathrm{\kappa B}$ activity. Leuk Lymphoma. 2011;52(12):2393-2395.

7. Specchia G, Pastore D, Carluccio P, et al. FLAG-IDA in the treatment of refractory/relapsed adult acute lymphoblastic leukemia. Ann Hematol. 2005;84(12):792-795.

8. Cortes J, Thomas D, Koller C, et al. Phase I study of bortezomib in refractory or relapsed acute leukemias. Clin Cancer Res. 2004;10(10): 3371-3376.

9. Attar EC, De Angelo DJ, Supko JG, et al. Phase I and pharmacokinetic study of bortezomib in combination with idarubicin and cytarabine in patients with acute myelogenous leukemia. Clin Cancer Res. 2008;14(5):1446-1454.

10. Faderl S, Rai K, Gribben J, et al. Phase II study of single-agent bortezomib for the treatment of patients with fludarabine-refractory B-cell chronic lymphocytic leukemia. Cancer. 2006;107(5):916-924.

11. Huguet F, Leguay T, Raffoux E, et al. Pediatric-inspired therapy in adults with Philadelphia chromosome-negative acute lymphoblastic leukemia: the GRAALL-2003 study. J Clin Oncol. 2009;27(6):911-918.

12. ChalandonY,Thomas X, Hayette S, et al. First Results of the GRAAPH-2005 Study in younger Adult Patients with De Novo Philadelphia Positive Acute Lymphoblastic Leukemia: 50th ASH Annual Meeting and Exposition, San Francisco, CA, USA, 6-9 December 2008. Blood. 2008;112(2).

13. Kantarjian HM, O'Brien S, Smith TL, et al. Results of treatment with hyper-CVAD, a dose-intensive regimen, in adult acute lymphocytic leukemia. J Clin Oncol. 2000;18(3):547-561.

\section{Dovepress}

\section{Publish your work in this journal}

The International Journal of General Medicine is an international, peer-reviewed open-access journal that focuses on general and internal medicine, pathogenesis, epidemiology, diagnosis, monitoring and treatment protocols. The journal is characterized by the rapid reporting of reviews, original research and clinical studies across all disease areas.
A key focus is the elucidation of disease processes and management protocols resulting in improved outcomes for the patient.The manuscript management system is completely online and includes a very quick and fair peer-review system. Visit http://www.dovepress.com/ testimonials.php to read real quotes from published authors. 